\title{
Modeling the Adaptive Immunity and Both Modes of Transmission in HIV Infection
}

\author{
Khalid Hattaf $1,2, *$ (iD and Noura Yousfi ${ }^{2}$ \\ 1 Centre Régional des Métiers de l'Education et de la Formation (CRMEF), 20340 Derb Ghalef, \\ Casablanca, Morocco \\ 2 Laboratory of Analysis, Modeling and Simulation (LAMS), Faculty of Sciences Ben M'sik, \\ Hassan II University, P.O. Box 7955, Sidi Othman, Casablanca, Morocco; nourayousfi@gmail.com \\ * Correspondence: k.hattaf@yahoo.fr; Tel.: +212-664407825
}

Received: 15 February 2018; Accepted: 4 May 2018; Published: 8 May 2018

\begin{abstract}
Human immunodeficiency virus (HIV) is a retrovirus that causes HIV infection and over time acquired immunodeficiency syndrome (AIDS). It can be spread and transmitted through two fundamental modes, one by virus-to-cell infection, and the other by direct cell-to-cell transmission. In this paper, we propose a new mathematical model that incorporates both modes of transmission and takes into account the role of the adaptive immune response in HIV infection. We first show that the proposed model is mathematically and biologically well posed. Moreover, we prove that the dynamical behavior of the model is fully determined by five threshold parameters. Furthermore, numerical simulations are presented to confirm our theoretical results.
\end{abstract}

Keywords: HIV infection; immunity; Lyapunov functional; global stability

\section{Introduction}

Viruses are very small infectious agents that need to penetrate inside a cell of their host to replicate and multiply. Several viruses attack the human body, such as influenza virus, human immunodeficiency virus (HIV), hepatitis B virus (HBV), hepatitis C virus (HCV), Ebola virus, Zika virus, and so on. Viral infections caused by these viruses represent a major global health problem by causing the mortality of millions of people and the expenditure of enormous amounts of money in health care and disease control. In this study, we are interested in the viral infection caused by HIV. The World Health Organization (WHO) estimates that 36.7 million people were living with HIV at the end of 2016, and more than 1 million people died from HIV-related causes in 2016 [1]. In Morocco, the number of people living with HIV is estimated at 28,740, and 1097 people died from AIDS in 2014, while the cumulative number of HIV / AIDS cases reported since the beginning of the epidemic is 10,017 [2].

Many mathematical models have been developed to better understand the dynamics of HIV infection. One of the earliest of these models was presented by Perelson et al. [3] in 1996. This model is given by the following system:

$$
\begin{cases}\dot{T}(t) & =\lambda-d T(t)-\beta_{1} T(t) V(t) \\ \dot{I}(t) & =\beta_{1} T(t) V(t)-a I(t) \\ \dot{V}(t) & =k I(t)-\mu V(t)\end{cases}
$$

where $T(t), I(t)$, and $V(t)$ are the concentrations of healthy $\mathrm{CD}^{+} \mathrm{T}$ cells, infected cells, and free virus at time $t$, respectively. Healthy cells are produced at rate $\lambda$, die at rate $d$, and become infected by free virus at rate $\beta_{1}$. The parameter $a$ is the death rate of infected cells. Free virus is produced by an infected cell at rate $k$ and is removed at rate $\mu$. 
In the system given by Equation (1), the cell infection is instantaneous and is caused only by contact with free virus. In reality, there are two kinds of delays: one in cell infection, and the other in virus production. In addition, HIV can spread by two fundamental modes, one by virus-to-cell infection, and the other by direct cell-to-cell transmission. For these above reasons, Lai and Zou [4] improved the model of Perelson et al. [3] by incorporating the two modes of transmission and infinite distributed delay in cell infection. They obtained the following model:

$$
\left\{\begin{array}{l}
\dot{T}(t)=\lambda-d T(t)-\beta_{1} T(t) V(t)-\beta_{2} T(t) I(t) \\
\dot{I}(t)=\int_{0}^{\infty} f_{1}(\tau) e^{-\alpha_{1} \tau}\left[\beta_{1} T(t-\tau) V(t-\tau)+\beta_{2} T(t-\tau) I(t-\tau)\right] d \tau-a I(t) \\
\dot{V}(t)=k I(t)-\mu V(t)
\end{array}\right.
$$

where $\beta_{2} T(t) I(t)$ denotes the rate for a target cell to contact with an infected cell. It is assumed that the virus or infected cell contacts an uninfected target cell at time $t-\tau$ and the cell becomes infected at time $t$, where $\tau$ is a random variable taken from a probability distribution $f_{1}(\tau)$. The term $e^{-\alpha_{1} \tau}$ represents the probability of surviving from time $t-\tau$ to time $t$, where $\alpha_{1}$ is the death rate for infected but not yet virus-producing cells. The other parameters have the same biological meaning as in Equation (1).

On the other hand, the adaptive immune responses of cytotoxic T lymphocytes (CTLs) and antibodies play an important role in the control of HIV infection. The first immune response exerted by CTL cells is called the cellular immunity. However, the second immune response mediated by antibodies is called the humoral immunity. In the literature, several authors are interested in modeling the role of these arms of immunity in viral infections. In 2016, Wang et al. [5] improved the model given by Equation (2) by considering the role of cellular immune response. In the same year, Elaiw et al. [6] improved the model given by Equation (2) by considering only the role of humoral immune response and infinite distributed delay in virus production. In 2017, Lin et al. [7] improved the models of Wang and Zou [8] and Murase et al. [9] by incorporating both modes of transmission, intracellular delay and humoral immunity.

The aim of this work is to improve and generalize all the above models by proposing a new mathematical model that takes into account the role of the adaptive immune response in HIV infection and incorporates both modes of transmission. To this end, the next section deals with the presentation of our model and some properties of solutions, such as positivity and boundedness. In Section 2, we derive the threshold parameters of our model and discuss the existence of equilibria. The global stability of equilibria is investigated in Section 3. Some numerical simulations of our main results are presented in Section 4. The mathematical and biological conclusions are given in Section 5.

\section{Presentation of the Model}

To model the role of the adaptive immune response in HIV infection with both virus-to-cell infection and cell-to-cell transmission, we propose the following model:

$$
\left\{\begin{array}{l}
\dot{T}(t)=\lambda-d T(t)-\beta_{1} T(t) V(t)-\beta_{2} T(t) I(t), \\
\dot{I}(t)=\int_{0}^{\infty} f_{1}(\tau) e^{-\alpha_{1} \tau}\left[\beta_{1} T(t-\tau) V(t-\tau)+\beta_{2} T(t-\tau) I(t-\tau)\right] d \tau \\
\quad-a I(t)-p I(t) Z(t), \\
\dot{V}(t)=k \int_{0}^{\infty} f_{2}(\tau) e^{-\alpha_{2} \tau} I(t-\tau) d \tau-\mu V(t)-q V(t) W(t), \\
\dot{W}(t)=g V(t) W(t)-h W(t), \\
\dot{Z}(t)=c I(t) Z(t)-b Z(t),
\end{array}\right.
$$

where $W(t)$ and $Z(t)$ are the concentrations of antibodies and CTL cells at time $t$, respectively. Free HIV particles are neutralized by the antibodies at rate $q V(t) W(t)$. However, the infected cells are killed by CTL cells at rate $p I(t) Z(t)$. Antibodies develop in response to free virus at rate $g V(t) W(t)$, and CTL cells expand in response to viral antigens derived from infected cells at rate $c I(t) Z(t)$. The parameters $h$ and $b$ are, respectively, the death rates of antibodies and CTL cells. Further, we assume that the time necessary for the newly produced virions to become mature and infectious is a random 
variable with a probability distribution $f_{2}(\tau)$. The term $e^{-\alpha_{2} \tau}$ denotes the probability of surviving the immature virions during the delay period, where $\frac{1}{\alpha_{2}}$ is the average lifetime of an immature virus. Therefore, the integral $\int_{0}^{\infty} f_{2}(\tau) e^{-\alpha_{2} \tau} I(t-\tau) d \tau$ describes the mature viral particles produced at time $t$. The other variables and parameters are defined as those in the systems given by Equations (1) and (2). In this section, we first investigate the nonnegativity and boundedness of solutions under the following nonnegative initial conditions:

$$
\begin{array}{ll}
T(\theta)=\phi_{1}(\theta) \geq 0, \quad I(\theta)=\phi_{2}(\theta) \geq 0, \quad V(\theta)=\phi_{3}(\theta) \geq 0, \\
W(\theta)=\phi_{4}(\theta) \geq 0, \quad Z(\theta)=\phi_{5}(\theta) \geq 0, \quad \theta \in(-\infty, 0] .
\end{array}
$$

We define the Banach space for the fading memory type as follows:

$$
\begin{aligned}
C_{\alpha}= & \left\{\varphi \in C\left((-\infty, 0], \mathbb{R}_{+}^{5}\right): \varphi(\theta) e^{\alpha \theta}\right. \text { is uniformly continuous } \\
& \text { on } \left.(-\infty, 0] \text { and }\|\varphi\|=\sup _{\theta \leq 0}|\varphi(\theta)| e^{\alpha \theta}<\infty\right\},
\end{aligned}
$$

where $\alpha$ is a positive constant and $\mathbb{R}_{+}^{5}=\left\{\left(x_{1}, \ldots, x_{5}\right): x_{i} \geq 0, i=1, \ldots, 5\right\}$.

Theorem 1. For any initial condition $\phi=\left(\phi_{1}, \phi_{2}, \phi_{3}, \phi_{4}, \phi_{5}\right) \in C_{\alpha}$ satisfying Equation (4), the model given by Equation (3) has a unique solution on $[0,+\infty)$. Furthermore, this solution is nonnegative and bounded for all $t \geq 0$.

Proof. By the fundamental theory of functional differential equations [10-12], the model given by Equation (3) with initial condition $\phi \in C_{\alpha}$ has a unique local solution on $\left[0, t_{\max }\right)$, where $t_{\max }$ is the maximal existence time for the solution of Equation (3).

First, we prove that $T(t)>0$ for all $t \in\left[0, t_{\max }\right)$. In fact, supposing the contrary, we let $t_{1}>0$ be the first time such that $T\left(t_{1}\right)=0$ and $\dot{T}\left(t_{1}\right) \leq 0$. By the first equation of the model given by Equation (3), we have $\dot{T}\left(t_{1}\right)=\lambda>0$, which is a contradiction. Thus, $T(t)>0$ for all $t \in\left[0, t_{\text {max }}\right)$. By Equation (3), we have

$$
\begin{aligned}
I(t)= & \phi_{2}(0) e^{-\int_{0}^{t}(a+p Z(s)) d s}+\int_{0}^{t} e^{-\int_{\xi}^{t}(a+p Z(s)) d s} \int_{0}^{\infty} f_{1}(\tau) e^{-\alpha_{1} \tau}\left[\beta_{1} T(\xi-\tau) V(\xi-\tau)\right. \\
& \left.+\beta_{2} T(\xi-\tau) I(\xi-\tau)\right] d \tau d \xi, \\
V(t)= & \phi_{3}(0) e^{-\int_{0}^{t}(\mu+q W(s)) d s}+k \int_{0}^{t} e^{-\int_{\tilde{\xi}}^{t}(\mu+q W(s)) d s} \int_{0}^{h_{2}} f_{2}(\tau) e^{-\alpha_{2} \tau} I(\xi-\tau) d \tau d \xi, \\
W(t)= & \phi_{4}(0) e^{\int_{0}^{t}(g V(s)-h) d s}, \\
Z(t)= & \phi_{5}(0) e^{\int_{0}^{t}(c I(s)-b) d s,}
\end{aligned}
$$

which implies that $I(t), V(t), W(t)$, and $Z(t)$ are nonnegative for all $t \in\left[0, t_{\text {max }}\right)$.

Next, we show the boundedness of each solution. From the first equation of the model given by Equation (3), we have $\dot{T}(t) \leq \lambda-d T(t)$, which implies that

$$
\limsup _{t \rightarrow+\infty} T(t) \leq \frac{\lambda}{d}
$$

Then $T(t)$ is bounded. Let

$$
G_{1}(t)=I(t)+\frac{p}{c} Z(t)+\int_{0}^{\infty} f_{1}(\tau) e^{-\alpha_{1} \tau} T(t-\tau) d \tau .
$$


Because $T(t)$ is bounded and $\int_{0}^{\infty} f_{1}(\tau) d \tau=1$, the integral in $G_{1}(t)$ is well defined and differentiable with respect to $t$. Hence,

$$
\begin{aligned}
\frac{d G_{1}(t)}{d t} & =\lambda \int_{0}^{\infty} f_{1}(\tau) e^{-\alpha_{1} \tau} d \tau-d \int_{0}^{\infty} f_{1}(\tau) e^{-\alpha_{1} \tau} T(t-\tau) d \tau-a I(t)-\frac{p b}{c} Z(t) \\
& \leq \lambda \eta_{1}-\delta_{1} G_{1}(t)
\end{aligned}
$$

where $\delta_{1}=\min \{a, b, d\}$ and

$$
\eta_{i}=\int_{0}^{\infty} f_{i}(\tau) e^{-\alpha_{i} \tau} d \tau, \quad i=1,2
$$

Thus, $G_{1}(t) \leq M:=\max \left\{G_{1}(0), \frac{\lambda \eta_{1}}{\delta_{1}}\right\}$, which implies that $I(t)$ and $Z(t)$ are bounded. It remains to prove that $V(t)$ and $W(t)$ are bounded. To this end, we consider

$$
G_{2}(t)=V(t)+\frac{q}{g} W(t)
$$

then

$$
\begin{aligned}
\frac{d G_{2}(t)}{d t} & =k \int_{0}^{\infty} f_{2}(\tau) e^{-\alpha_{2} \tau} I(t-\tau) d \tau-\mu V(t)-\frac{h q}{g} W(t) \\
& \leq k M \eta_{2}-\delta_{2} G_{2}(t)
\end{aligned}
$$

where $\delta_{2}=\min \{\mu, h\}$. Similarly to the above, we deduce that $V(t)$ and $W(t)$ are also bounded. We have proved that all variables of Equation (3) are bounded, which implies that $t_{\text {max }}=+\infty$ and that the solution exists globally.

If in addition to Equation (4), we suppose that $\phi_{i}(0)>0$ for all $i=1, \ldots, 5$; then we obtain the following remark.

Remark 1. If $\phi=\left(\phi_{1}, \phi_{2}, \phi_{3}, \phi_{4}, \phi_{5}\right) \in C_{\alpha}$ satisfies Equation (4) with $\phi_{i}(0)>0$, then each solution of Equation (3) with initial condition $\phi$ remains positive and bounded for all $t \geq 0$.

Next, we derive threshold numbers and identify biological equilibria for the model given by Equation (3). Clearly, Equation (3) always has an infection-free equilibrium of the form $E_{0}\left(T_{0}, 0,0,0,0\right)$, where $T_{0}=\frac{\lambda}{d}$. Therefore, the basic reproduction number of Equation (3) can be defined as

$$
R_{0}=\frac{\beta_{1} k \lambda \eta_{1} \eta_{2}+\beta_{2} \lambda \mu \eta_{1}}{d a \mu}
$$

As in [13], $R_{0}$ can be rewritten as $R_{0}=R_{01}+R_{02}$, where $R_{01}=\frac{\beta_{1} k \lambda \eta_{1} \eta_{2}}{d a \mu}$ is the basic reproduction number corresponding to the virus-to-cell infection mode, and $R_{02}=\frac{\beta_{2} \lambda \eta_{1}}{d a}$ is the basic reproduction number corresponding to the cell-to-cell transmission mode.

When $R_{0}>1$, Equation (3) has another infection equilibrium without immunity, $E_{1}\left(T_{1}, I_{1}, V_{1}, 0,0\right)$, where

$$
T_{1}=\frac{\lambda}{d R_{0}}, I_{1}=\frac{d \mu\left(R_{0}-1\right)}{\beta_{1} k \eta_{2}+\beta_{2} \mu} \text { and } V_{1}=\frac{k d \eta_{2}\left(R_{0}-1\right)}{\beta_{1} k \eta_{2}+\beta_{2} \mu}
$$


If both humoral and cellular immune responses have not been established, we have $g V_{1}-h \leq 0$ and $c I_{1}-b \leq 0$. Thus, we define the reproduction number for humoral immunity:

$$
R_{1}^{W}=\frac{g V_{1}}{h}=\frac{g k d \eta_{2}\left(R_{0}-1\right)}{h\left(\beta_{1} k \eta_{2}+\beta_{2} \mu\right)}
$$

and the reproduction number for cellular immunity:

$$
R_{1}^{Z}=\frac{c I_{1}}{b}=\frac{c d \mu\left(R_{0}-1\right)}{b\left(\beta_{1} k \eta_{2}+\beta_{2} \mu\right)} .
$$

Hence, $g V_{1}-h \leq 0$ and $c I_{1}-b \leq 0$ are equivalent to $R_{1}^{W} \leq 1$ and $R_{1}^{Z} \leq 1$, respectively.

When $R_{1}^{W}>1$, Equation (3) has an infection equilibrium with only humoral immunity, $E_{2}\left(T_{2}, I_{2}, V_{2}, W_{2}, 0\right)$, where

$$
T_{2}=\frac{a I_{2}}{\eta_{1}\left(\beta_{1} V_{2}+\beta_{2} I_{2}\right)}, I_{2}=\frac{-B+\sqrt{B^{2}+4 A C}}{2 A}, V_{2}=\frac{h}{g}, W_{2}=\frac{\mu}{q}\left(\frac{k \eta_{2} I_{2}}{\mu V_{2}}-1\right),
$$

with $A=a g \beta_{2}, B=a\left(d g+h \beta_{1}\right)-\lambda \eta_{1} \beta_{2} g$, and $C=\lambda h \eta_{1} \beta_{1}$.

It is not hard to see that $T_{2}, I_{2}$ and $V_{2}$ are positive. It remains to check that $W_{2}$ is positive. Because $R_{1}^{W}>1$ and

$$
\frac{\mu V_{2}}{k^{2} \eta_{2}^{2}}\left(A \mu V_{2}+B\right)-C=\frac{\mu a h V_{2}}{k^{2} \eta_{2}^{2}}\left(1-R_{1}^{W}\right)
$$

we easily deduce that $W_{2}>0$. If cellular immunity has not been established, we have $c I_{2}-b \leq 0$. For this, we define the reproduction number for cellular immunity in competition as

$$
R_{2}^{Z}=\frac{c I_{2}}{b}
$$

which implies that $c I_{2}-b \leq 0$ is equivalent to $R_{2}^{Z} \leq 1$.

When $R_{1}^{Z}>1$, Equation (3) has an infection equilibrium with only cellular immunity, $E_{3}\left(T_{3}, I_{3}, V_{3}, 0, Z_{3}\right)$, where

$$
T_{3}=\frac{\lambda c \mu}{d c \mu+b\left(k \beta_{1} \eta_{2}+\mu \beta_{2}\right)}, I_{3}=\frac{b}{c}, V_{3}=\frac{k b \eta_{2}}{c \mu}, Z_{3}=\frac{a}{p}\left(\frac{R_{0}}{1+\frac{a b}{\lambda c \eta_{1}} R_{0}}-1\right)
$$

We have that $T_{3}, I_{3}$, and $V_{3}$ are positive. It suffices to check that $Z_{3}$ is positive. Because $R_{1}^{Z}>1$ and

$$
\frac{R_{0}}{1+\frac{a b}{\lambda c \eta_{1}} R_{0}}-1=\frac{b\left(k \beta_{1} \eta_{2}+\mu \beta_{2}\right)}{d c \mu\left(1+\frac{a b}{\lambda c \eta_{1}} R_{0}\right)}\left(R_{1}^{Z}-1\right),
$$

we deduce that $Z_{3}>0$. If humoral immunity has not been established, we have $g V_{3}-h \leq 0$. In this case, we define the reproduction number for humoral immunity in competition as

$$
R_{3}^{W}=\frac{g V_{3}}{h}
$$

which implies that $g V_{3}-h \leq 0$ is equivalent to $R_{3}^{W} \leq 1$.

When $R_{2}^{Z}>1$ and $R_{3}^{W}>1$, Equation (3) has an infection equilibrium with both cellular and humoral immune responses, $E_{4}\left(T_{4}, I_{4}, V_{4}, W_{4}, Z_{4}\right)$, where

$$
T_{4}=\frac{\lambda g c}{d g c+\beta_{1} h c+\beta_{2} b g}, I_{4}=\frac{b}{c}, V_{4}=\frac{h}{g}, W_{4}=\frac{\mu}{q}\left(R_{3}^{W}-1\right), Z_{4}=\frac{a}{p}\left(\frac{\lambda c \eta_{1}\left(\beta_{1} h c+\beta_{2} b g\right)}{a b\left(d g c+\beta_{1} h c+\beta_{2} b g\right)}-1\right) .
$$


We have $T_{4}>0, I_{4}>0, V_{4}>0$, and $W_{4}>0\left(\right.$ as $\left.R_{3}^{W}>1\right)$. It remains to check that $Z_{4}>1$. On the other hand, $R_{2}^{Z}>1$ is equivalent to $C>\frac{b}{c^{2}}(b A+c B)$. By a simple computation, we have

$$
C-\frac{b}{c^{2}}(b A+c B)=\frac{p b}{c^{2}}\left(d g c+\beta_{1} h c+\beta_{2} b g\right) Z_{4} .
$$

Summarizing the above discussions, we obtain the following theorem.

\section{Theorem 2.}

(i) If $R_{0} \leq 1$, then Equation (3) always has one infection-free equilibrium, $E_{0}\left(T_{0}, 0,0,0,0\right)$, where $T_{0}=\frac{\lambda}{d}$.

(ii) If $R_{0}>1$, then Equation (3) has an infection equilibrium without immunity, $E_{1}\left(T_{1}, I_{1}, V_{1}, 0,0\right)$, where

$$
T_{1}=\frac{\lambda}{d R_{0}}, I_{1}=\frac{d \mu\left(R_{0}-1\right)}{\beta_{1} k \eta_{2}+\beta_{2} \mu} \text { and } V_{1}=\frac{k d \eta_{2}\left(R_{0}-1\right)}{\beta_{1} k \eta_{2}+\beta_{2} \mu} .
$$

(iii) If $R_{1}^{W}>1$, then Equation (3) has an infection equilibrium with only humoral immunity, $E_{2}\left(T_{2}, I_{2}, V_{2}, W_{2}, 0\right)$, where

$$
T_{2}=\frac{a I_{2}}{\eta_{1}\left(\beta_{1} V_{2}+\beta_{2} I_{2}\right)}, I_{2}=\frac{-B+\sqrt{B^{2}+4 A C}}{2 A}, V_{2}=\frac{h}{g}, W_{2}=\frac{\mu}{q}\left(\frac{k \eta_{2} I_{2}}{\mu V_{2}}-1\right) .
$$

(iv) If $R_{1}^{Z}>1$, then Equation (3) has an infection equilibrium with only cellular immunity, $E_{3}\left(T_{3}, I_{3}, V_{3}, 0, Z_{3}\right)$, where

$$
T_{3}=\frac{\lambda c \mu}{d c \mu+b\left(k \beta_{1} \eta_{2}+\mu \beta_{2}\right)}, I_{3}=\frac{b}{c}, V_{3}=\frac{k b \eta_{2}}{c \mu}, Z_{3}=\frac{a}{p}\left(\frac{R_{0}}{1+\frac{a b}{\lambda c \eta_{1}} R_{0}}-1\right) .
$$

(v) If $R_{2}^{Z}>1$ and $R_{3}^{W}>1$, then Equation (3) has an infection equilibrium with both cellular and humoral immune responses, $E_{4}\left(T_{4}, I_{4}, V_{4}, W_{4}, Z_{4}\right)$, where

$$
\begin{gathered}
T_{4}=\frac{\lambda g c}{d g c+\beta_{1} h c+\beta_{2} b g}, I_{4}=\frac{b}{c}, V_{4}=\frac{h}{g}, W_{4}=\frac{\mu}{q}\left(R_{3}^{W}-1\right) \text { and } \\
Z_{4}=\frac{a}{p}\left(\frac{\lambda c \eta_{1}\left(\beta_{1} h c+\beta_{2} b g\right)}{a b\left(d g c+\beta_{1} h c+\beta_{2} b g\right)}-1\right) .
\end{gathered}
$$

It is important to note that

$$
R_{3}^{W}=\frac{R_{1}^{W}}{R_{1}^{Z}}=\frac{g k b \eta_{2}}{h \mu c}, W_{2}=\frac{\mu}{q}\left(R_{2}^{Z} R_{3}^{W}-1\right) \text { and } R_{3}^{W}>\frac{1}{R_{2}^{Z}}
$$

\section{Global Stability}

In this section, we investigate the global stability of the five equilibria of Equation (3) by constructing appropriate Lyapunov functionals. We first analyze the global stability of the infection-free equilibrium.

Theorem 3. The infection-free equilibrium $E_{0}$ is globally asymptotically stable when $R_{0} \leq 1$. 
Proof. To study the global stability of $E_{0}$, we consider a Lyapunov functional defined as follows:

$$
\begin{aligned}
L_{0}(t)= & T_{0} \Phi\left(\frac{T(t)}{T_{0}}\right)+\frac{1}{\eta_{1}} I(t)+\frac{\beta_{1} T_{0}}{\mu} V(t)+\frac{q \beta_{1} T_{0}}{g \mu} W(t)+\frac{p}{c \eta_{1}} Z(t) \\
& +\frac{1}{\eta_{1}} \int_{0}^{\infty} f_{1}(\tau) e^{-\alpha_{1} \tau} \int_{t-\tau}^{t}\left[\beta_{1} T(s) V(s)+\beta_{2} T(s) I(s)\right] d s d \tau \\
& +\frac{k \beta_{1} T_{0}}{\mu} \int_{0}^{\infty} f_{2}(\tau) e^{-\alpha_{2} \tau} \int_{t-\tau}^{t} I(s) d s d \tau,
\end{aligned}
$$

where $\Phi(x)=x-1-\ln x$ for $x>0$. It is not hard to see that $\Phi(x) \geq 0$ for all $x \in(0,+\infty)$. Hence, the functional $L_{0}$ is nonnegative.

In order to simplify the presentation, we use the following notations: $\Psi=\Psi(t)$ and $\Psi_{\tau}=\Psi(t-\tau)$ for any $\Psi \in\{T, I, V, W, Z\}$. Calculating the time derivative of $L_{0}$ along the positive solution of Equation (3), we obtain

$$
\begin{aligned}
\left.\frac{d L_{0}}{d t}\right|_{(3)}= & \left(1-\frac{T_{0}}{T}\right) \dot{T}+\frac{1}{\eta_{1}} \dot{I}+\frac{\beta_{1} T_{0}}{\mu} \dot{V}+\frac{q \beta_{1} T_{0}}{g \mu} \dot{W}+\frac{p}{c \eta_{1}} \dot{Z} \\
& +\frac{1}{\eta_{1}} \int_{0}^{\infty} f_{1}(\tau) e^{-\alpha_{1} \tau}\left[\beta_{1} T V+\beta_{2} T I-\beta_{1} T_{\tau} V_{\tau}-\beta_{2} T_{\tau} I_{\tau}\right] d \tau \\
& +\frac{k \beta_{1} T_{0}}{\mu} \int_{0}^{\infty} f_{2}(\tau) e^{-\alpha_{2} \tau}\left(I-I_{\tau}\right) d \tau \\
= & -\frac{d}{T}\left(T-T_{0}\right)^{2}+\frac{a}{\eta_{1}}\left(R_{0}-1\right) I-\frac{h q \beta_{1} T_{0}}{g \mu} W-\frac{p}{c \eta_{1}} Z .
\end{aligned}
$$

If follows from $R_{0} \leq 1$ that $\frac{d L_{0}}{d t} \leq 0$. It is straightforward to show that the largest invariant set in $\left\{(T, I, V, W, Z) \mid \frac{d L_{0}}{d t}=0\right\}$ is $\left\{E_{0}\right\}$. By LaSalle's invariance principle [14], the infection-free equilibrium $E_{0}$ is globally asymptotically stable when $R_{0} \leq 1$.

When $R_{0}>1$, Equation (3) has four infection steady states $E_{i}, 1 \leq i \leq 4$. The following theorem characterizes the global stability of these steady states.

Theorem 4. Assume $R_{0}>1$.

(i) The infection equilibrium without immunity $E_{1}$ is globally asymptotically stable if $R_{1}^{W} \leq 1$ and $R_{1}^{Z} \leq 1$.

(ii) The infection equilibrium with only humoral immunity $E_{2}$ is globally asymptotically stable if $R_{1}^{W}>1$ and $R_{2}^{Z} \leq 1$.

(iii) The infection equilibrium with only cellular immunity $E_{3}$ is globally asymptotically stable if $R_{1}^{Z}>1$ and $R_{3}^{W} \leq 1$.

(iv) The infection equilibrium with both cellular and humoral immune responses $E_{4}$ is globally asymptotically stable if $R_{2}^{Z}>1$ and $R_{3}^{W}>1$.

Proof. For (i), consider the following Lyapunov functional:

$$
\begin{aligned}
L_{1}(t)= & T_{1} \Phi\left(\frac{T(t)}{T_{1}}\right)+\frac{1}{\eta_{1}} I_{1} \Phi\left(\frac{I(t)}{I_{1}}\right)+\frac{\beta_{1} T_{1} V_{1}}{k \eta_{2} I_{1}} V_{1} \Phi\left(\frac{V(t)}{V_{1}}\right)+\frac{q \beta_{1} T_{1}}{g \mu} W(t)+\frac{p}{c \eta_{1}} Z(t) \\
& +\frac{\beta_{1} T_{1} V_{1}}{\eta_{1}} \int_{0}^{\infty} f_{1}(\tau) e^{-\alpha_{1} \tau} \int_{t-\tau}^{t} \Phi\left(\frac{T(s) V(s)}{T_{1} V_{1}}\right) d s d \tau \\
& +\frac{\beta_{2} T_{1} I_{1}}{\eta_{1}} \int_{0}^{\infty} f_{1}(\tau) e^{-\alpha_{1} \tau} \int_{t-\tau}^{t} \Phi\left(\frac{T(s) I(s)}{T_{1} I_{1}}\right) d s d \tau \\
& +\frac{\beta_{1} T_{1} V_{1}}{\eta_{2}} \int_{0}^{\infty} f_{2}(\tau) e^{-\alpha_{2} \tau} \int_{t-\tau}^{t} \Phi\left(\frac{I(s)}{I_{1}}\right) d s d \tau .
\end{aligned}
$$


Then

$$
\begin{aligned}
\left.\frac{d L_{1}}{d t}\right|_{(3)}= & \left(1-\frac{T_{1}}{T}\right) \dot{T}+\frac{1}{\eta_{1}}\left(1-\frac{I_{1}}{I}\right) \dot{I}+\frac{\beta_{1} T_{1} V_{1}}{k \eta_{2} I_{1}}\left(1-\frac{V_{1}}{V}\right) \dot{V}+\frac{q \beta_{1} T_{1}}{g \mu} \dot{W}+\frac{p}{c \eta_{1}} \dot{Z} \\
& +\frac{\beta_{1} T_{1} V_{1}}{\eta_{1}} \int_{0}^{\infty} f_{1}(\tau) e^{-\alpha_{1} \tau}\left(\Phi\left(\frac{T V}{T_{1} V_{1}}\right)-\Phi\left(\frac{T_{\tau} V_{\tau}}{T_{1} V_{1}}\right)\right) d \tau \\
& +\frac{\beta_{2} T_{1} I_{1}}{\eta_{1}} \int_{0}^{\infty} f_{1}(\tau) e^{-\alpha_{1} \tau}\left(\Phi\left(\frac{T I}{T_{1} I_{1}}\right)-\Phi\left(\frac{T_{\tau} I_{\tau}}{T_{1} I_{1}}\right)\right) d \tau \\
& +\frac{\beta_{1} T_{1} V_{1}}{\eta_{2}} \int_{0}^{\infty} f_{2}(\tau) e^{-\alpha_{1} \tau}\left(\Phi\left(\frac{I}{I_{1}}\right)-\Phi\left(\frac{I_{\tau}}{I_{1}}\right)\right) d \tau .
\end{aligned}
$$

By $\lambda=d T_{1}+\beta_{1} T_{1} V_{1}+\beta_{2} T_{1} I_{1}=d T_{1}+\frac{a}{\eta_{1}} I_{1}$ and $k \eta_{2} I_{1}=\mu V_{1}$, we obtain

$$
\begin{aligned}
\left.\frac{d L_{1}}{d t}\right|_{(3)}= & -\frac{d}{T}\left(T-T_{1}\right)^{2}+\frac{h q \beta_{1} T_{1}}{g \mu}\left(R_{1}^{W}-1\right) W+\frac{p b}{c \eta_{1}}\left(R_{1}^{Z}-1\right) Z \\
& +\frac{\beta_{1} T_{1} V_{1}}{\eta_{1}} \int_{0}^{\infty} f_{1}(\tau) e^{-\alpha_{1} \tau}\left[3-\frac{T_{1}}{T}-\frac{T_{\tau} V_{\tau} I_{1}}{T_{1} V_{1} I}+\ln \left(\frac{T_{\tau} V_{\tau}}{T V}\right)\right] d \tau \\
& +\frac{\beta_{2} T_{1} I_{1}}{\eta_{1}} \int_{0}^{\infty} f_{1}(\tau) e^{-\alpha_{1} \tau}\left[2-\frac{T_{1}}{T}-\frac{T_{\tau} I_{\tau}}{T_{1} I}+\ln \left(\frac{T_{\tau} I_{\tau}}{T I}\right)\right] d \tau \\
& -\frac{\beta_{1} T_{1} V_{1}}{\eta_{2}} \int_{0}^{\infty} f_{2}(\tau) e^{-\alpha_{2} \tau}\left[\frac{V_{1} I_{\tau}}{V I_{1}}-\ln \left(\frac{I_{\tau}}{I}\right)\right] d \tau .
\end{aligned}
$$

Thus,

$$
\begin{aligned}
\left.\frac{d L_{1}}{d t}\right|_{(3)}= & -\frac{d}{T}\left(T-T_{1}\right)^{2}+\frac{h q \beta_{1} T_{1}}{g \mu}\left(R_{1}^{W}-1\right) W+\frac{p b}{c \eta_{1}}\left(R_{1}^{Z}-1\right) Z \\
& -\frac{\beta_{1} T_{1} V_{1}}{\eta_{1}} \int_{0}^{\infty} f_{1}(\tau) e^{-\alpha_{1} \tau}\left[\Phi\left(\frac{T_{1}}{T}\right)+\Phi\left(\frac{T_{\tau} V_{\tau} I_{1}}{T_{1} V_{1} I}\right)\right] d \tau \\
& -\frac{\beta_{2} T_{1} I_{1}}{\eta_{1}} \int_{0}^{\infty} f_{1}(\tau) e^{-\alpha_{1} \tau}\left[\Phi\left(\frac{T_{1}}{T}\right)+\Phi\left(\frac{T_{\tau} I_{\tau}}{T_{1} I}\right)\right] d \tau \\
& -\frac{\beta_{1} T_{1} V_{1}}{\eta_{2}} \int_{0}^{\infty} f_{2}(\tau) e^{-\alpha_{2} \tau} \Phi\left(\frac{V_{1} I_{\tau}}{V I_{1}}\right) d \tau .
\end{aligned}
$$

Because $\Phi(x) \geq 0$ for $x>0, R_{1}^{W} \leq 1$, and $R_{1}^{Z} \leq 1$, we have $\left.\frac{d L_{1}}{d t}\right|_{(3)} \leq 0$ with equality if and only if $T=T_{1}, I=I_{1}, V=V_{1}, W=0$, and $Z=0$. It follows from LaSalle's invariance principle that $E_{1}$ is globally asymptotically stable.

For (ii), consider the following Lyapunov functional:

$$
\begin{aligned}
L_{2}(t)= & T_{2} \Phi\left(\frac{T(t)}{T_{2}}\right)+\frac{1}{\eta_{1}} I_{2} \Phi\left(\frac{I(t)}{I_{2}}\right)+\frac{\beta_{1} T_{2} V_{2}}{k \eta_{2} I_{2}} V_{2} \Phi\left(\frac{V(t)}{V_{2}}\right) \\
& +\frac{q \beta_{1} T_{2} V_{2}}{g k \eta_{2} I_{2}} W_{2} \Phi\left(\frac{W(t)}{W_{2}}\right)+\frac{p}{c \eta_{1}} Z(t) \\
& +\frac{\beta_{1} T_{2} V_{2}}{\eta_{1}} \int_{0}^{\infty} f_{1}(\tau) e^{-\alpha_{1} \tau} \int_{t-\tau}^{t} \Phi\left(\frac{T(s) V(s)}{T_{2} V_{2}}\right) d s d \tau \\
& +\frac{\beta_{2} T_{2} I_{2}}{\eta_{1}} \int_{0}^{\infty} f_{1}(\tau) e^{-\alpha_{1} \tau} \int_{t-\tau}^{t} \Phi\left(\frac{T(s) I(s)}{T_{2} I_{2}}\right) d s d \tau \\
& +\frac{\beta_{1} T_{2} V_{2}}{\eta_{2}} \int_{0}^{\infty} f_{2}(\tau) e^{-\alpha_{2} \tau} \int_{t-\tau}^{t} \Phi\left(\frac{I(s)}{I_{2}}\right) d s d \tau
\end{aligned}
$$


Hence,

$$
\begin{aligned}
\left.\frac{d L_{2}}{d t}\right|_{(3)}= & \left(1-\frac{T_{2}}{T}\right) \dot{T}+\frac{1}{\eta_{1}}\left(1-\frac{I_{2}}{I}\right) \dot{I}+\frac{\beta_{1} T_{2} V_{2}}{k \eta_{2} I_{2}}\left(1-\frac{V_{2}}{V}\right) \dot{V} \\
& +\frac{q \beta_{1} T_{2} V_{2}}{g k \eta_{2} I_{2}}\left(1-\frac{W_{2}}{W}\right) \dot{W}+\frac{p}{c \eta_{1}} \dot{Z} \\
& +\frac{\beta_{1} T_{1} V_{1}}{\eta_{1}} \int_{0}^{\infty} f_{1}(\tau) e^{-\alpha_{1} \tau}\left(\Phi\left(\frac{T V}{T_{1} V_{1}}\right)-\Phi\left(\frac{T_{\tau} V_{\tau}}{T_{1} V_{1}}\right)\right) d \tau \\
& +\frac{\beta_{2} T_{1} I_{1}}{\eta_{1}} \int_{0}^{\infty} f_{1}(\tau) e^{-\alpha_{1} \tau}\left(\Phi\left(\frac{T I}{T_{1} I_{1}}\right)-\Phi\left(\frac{T_{\tau} I_{\tau}}{T_{1} I_{1}}\right)\right) d \tau \\
& +\frac{\beta_{1} T_{1} V_{1}}{\eta_{2}} \int_{0}^{\infty} f_{2}(\tau) e^{-\alpha_{1} \tau}\left(\Phi\left(\frac{I}{I_{1}}\right)-\Phi\left(\frac{I_{\tau}}{I_{1}}\right)\right) d \tau .
\end{aligned}
$$

By $\lambda=d T_{2}+\beta_{1} T_{2} V_{2}+\beta_{2} T_{2} I_{2}=d T_{2}+\frac{a}{\eta_{1}} I_{2}, V_{2}=\frac{h}{g}$, and $k \eta_{2} I_{2}=\mu V_{2}+q W_{2} V_{2}$, we obtain

$$
\begin{aligned}
\left.\frac{d L_{2}}{d t}\right|_{(3)}= & -\frac{d}{T}\left(T-T_{2}\right)^{2}+\frac{p b}{c \eta_{1}}\left(R_{2}^{Z}-1\right) Z \\
& +\frac{\beta_{1} T_{2} V_{2}}{\eta_{1}} \int_{0}^{\infty} f_{1}(\tau) e^{-\alpha_{1} \tau}\left[3-\frac{T_{2}}{T}-\frac{T_{\tau} V_{\tau} I_{2}}{T_{2} V_{2} I}+\ln \left(\frac{T_{\tau} V_{\tau}}{T V}\right)\right] d \tau \\
& +\frac{\beta_{2} T_{2} I_{2}}{\eta_{1}} \int_{0}^{\infty} f_{1}(\tau) e^{-\alpha_{1} \tau}\left[2-\frac{T_{2}}{T}-\frac{T_{\tau} I_{\tau}}{T_{2} I}+\ln \left(\frac{T_{\tau} I_{\tau}}{T I}\right)\right] d \tau \\
& -\frac{\beta_{1} T_{2} V_{2}}{\eta_{2}} \int_{0}^{\infty} f_{2}(\tau) e^{-\alpha_{2} \tau}\left[\frac{V_{2} I_{\tau}}{V I_{2}}-\ln \left(\frac{I_{\tau}}{I}\right)\right] d \tau .
\end{aligned}
$$

Thus,

$$
\begin{aligned}
\left.\frac{d L_{2}}{d t}\right|_{(3)}= & -\frac{d}{T}\left(T-T_{2}\right)^{2}+\frac{p b}{c \eta_{1}}\left(R_{2}^{Z}-1\right) Z \\
& -\frac{\beta_{1} T_{2} V_{2}}{\eta_{1}} \int_{0}^{\infty} f_{1}(\tau) e^{-\alpha_{1} \tau}\left[\Phi\left(\frac{T_{2}}{T}\right)+\Phi\left(\frac{T_{\tau} V_{\tau} I_{2}}{T_{2} V_{2} I}\right)\right] d \tau \\
& -\frac{\beta_{2} T_{2} I_{2}}{\eta_{1}} \int_{0}^{\infty} f_{1}(\tau) e^{-\alpha_{1} \tau}\left[\Phi\left(\frac{T_{2}}{T}\right)+\Phi\left(\frac{T_{\tau} I_{\tau}}{T_{2} I}\right)\right] d \tau \\
& -\frac{\beta_{1} T_{2} V_{2}}{\eta_{2}} \int_{0}^{\infty} f_{2}(\tau) e^{-\alpha_{2} \tau} \Phi\left(\frac{V_{2} I_{\tau}}{V I_{2}}\right) d \tau
\end{aligned}
$$

Because $R_{2}^{Z} \leq 1$ and $\Phi(x) \geq 0$ for $x>0$, we have $\left.\frac{d L_{2}}{d t}\right|_{(3)} \leq 0$ with equality if and only if $T=T_{2}$, $I=I_{2}$, and $V=V_{2}$. Then $\dot{I}=0$ and $\dot{V}=0$, which leads to $Z=0$ and $W=W_{2}$. Therefore, the largest compact invariant set in $\left\{(T, I, V, W, Z) \mid \frac{d L_{2}}{d t}=0\right\}$ is the singleton $\left\{E_{2}\right\}$, and the proof of (ii) is completed. 
For (iii), consider the following Lyapunov functional:

$$
\begin{aligned}
L_{3}(t)= & T_{3} \Phi\left(\frac{T(t)}{T_{3}}\right)+\frac{1}{\eta_{1}} I_{3} \Phi\left(\frac{I(t)}{I_{3}}\right)+\frac{\beta_{1} T_{3} V_{3}}{k \eta_{2} I_{3}} V_{3} \Phi\left(\frac{V(t)}{V_{3}}\right) \\
& +\frac{q \beta_{1} T_{3}}{g \mu} W(t)+\frac{p}{c \eta_{1}} Z_{2} \Phi\left(\frac{Z(t)}{Z_{3}}\right) \\
& +\frac{\beta_{1} T_{3} V_{3}}{\eta_{1}} \int_{0}^{\infty} f_{1}(\tau) e^{-\alpha_{1} \tau} \int_{t-\tau}^{t} \Phi\left(\frac{T(s) V(s)}{T_{3} V_{3}}\right) d s d \tau \\
& +\frac{\beta_{2} T_{3} I_{3}}{\eta_{1}} \int_{0}^{\infty} f_{1}(\tau) e^{-\alpha_{1} \tau} \int_{t-\tau}^{t} \Phi\left(\frac{T(s) I(s)}{T_{3} I_{3}}\right) d s d \tau \\
& +\frac{\beta_{1} T_{3} V_{3}}{\eta_{2}} \int_{0}^{\infty} f_{2}(\tau) e^{-\alpha_{2} \tau} \int_{t-\tau}^{t} \Phi\left(\frac{I(s)}{I_{3}}\right) d s d \tau
\end{aligned}
$$

From $\lambda=d T_{3}+\beta_{1} T_{3} V_{3}+\beta_{2} T_{3} I_{3}=d T_{3}+\frac{a}{\eta_{1}} I_{2}+\frac{p}{\eta_{1}} I_{3} Z_{3}, I_{3}=\frac{b}{c}$, and $k \eta_{2} I_{3}=\mu V_{3}$, we easily have

$$
\begin{aligned}
\left.\frac{d L_{3}}{d t}\right|_{(3)}= & -\frac{d}{T}\left(T-T_{3}\right)^{2}+\frac{h q \beta_{1} T_{3}}{g \mu}\left(R_{3}^{W}-1\right) W \\
& -\frac{\beta_{1} T_{3} V_{3}}{\eta_{1}} \int_{0}^{\infty} f_{1}(\tau) e^{-\alpha_{1} \tau}\left[\Phi\left(\frac{T_{3}}{T}\right)+\Phi\left(\frac{T_{\tau} V_{\tau} I_{3}}{T_{3} V_{3} I}\right)\right] d \tau \\
& -\frac{\beta_{2} T_{3} I_{3}}{\eta_{1}} \int_{0}^{\infty} f_{1}(\tau) e^{-\alpha_{1} \tau}\left[\Phi\left(\frac{T_{3}}{T}\right)+\Phi\left(\frac{T_{\tau} I_{\tau}}{T_{3} I}\right)\right] d \tau \\
& -\frac{\beta_{1} T_{3} V_{3}}{\eta_{2}} \int_{0}^{\infty} f_{2}(\tau) e^{-\alpha_{2} \tau} \Phi\left(\frac{V_{3} I_{\tau}}{V I_{3}}\right) d \tau .
\end{aligned}
$$

Consequently, $\left.\frac{d L_{3}}{d t}\right|_{(3)} \leq 0$ with equality if and only if $T=T_{3}, I=I_{3}$, and $V=V_{3}$. It follows from $\dot{I}=0$ and $\dot{V}=0$ that $Z=Z_{3}$ and $W=0$. By LaSalle's invariance principle, we deduce that $E_{3}$ is globally asymptotically stable.

Finally, we show (iv) by considering the following Lyapunov functional:

$$
\begin{aligned}
L_{4}(t)= & T_{4} \Phi\left(\frac{T(t)}{T_{4}}\right)+\frac{1}{\eta_{1}} I_{4} \Phi\left(\frac{I(t)}{I_{4}}\right)+\frac{\beta_{1} T_{4} V_{4}}{k \eta_{2} I_{4}} V_{4} \Phi\left(\frac{V(t)}{V_{4}}\right) \\
& +\frac{q \beta_{1} T_{4} V_{4}}{g k \eta_{2} I_{4}} W_{4} \Phi\left(\frac{W(t)}{W_{4}}\right)+\frac{p}{c \eta_{1}} Z_{4} \Phi\left(\frac{Z(t)}{Z_{4}}\right) \\
& +\frac{\beta_{1} T_{4} V_{4}}{\eta_{1}} \int_{0}^{\infty} f_{1}(\tau) e^{-\alpha_{1} \tau} \int_{t-\tau}^{t} \Phi\left(\frac{T(s) V(s)}{T_{4} V_{4}}\right) d s d \tau \\
& +\frac{\beta_{2} T_{4} I_{4}}{\eta_{1}} \int_{0}^{\infty} f_{1}(\tau) e^{-\alpha_{1} \tau} \int_{t-\tau}^{t} \Phi\left(\frac{T(s) I(s)}{T_{4} I_{4}}\right) d s d \tau \\
& +\frac{\beta_{1} T_{4} V_{4}}{\eta_{2}} \int_{0}^{\infty} f_{2}(\tau) e^{-\alpha_{2} \tau} \int_{t-\tau}^{t} \Phi\left(\frac{I(s)}{I_{4}}\right) d s d \tau .
\end{aligned}
$$

By $\lambda=d T_{4}+\beta_{1} T_{4} V_{4}+\beta_{2} T_{4} I_{4}=d T_{4}+\frac{a}{\eta_{1}} I_{4}+\frac{p}{\eta_{1}} I_{4} Z_{4}, I_{4}=\frac{b}{c} V_{4}=\frac{h}{g}$, and $k \eta_{2} I_{4}=\left(\mu+q W_{4}\right) V_{4}$, we obtain

$$
\begin{aligned}
\left.\frac{d L_{4}}{d t}\right|_{(3)}= & -\frac{d}{T}\left(T-T_{4}\right)^{2}+\frac{\beta_{1} T_{4} V_{4}}{\eta_{1}} \int_{0}^{\infty} f_{1}(\tau) e^{-\alpha_{1} \tau}\left[3-\frac{T_{4}}{T}-\frac{T_{\tau} V_{\tau} I_{4}}{T_{4} V_{4} I}+\ln \left(\frac{T_{\tau} V_{\tau}}{T V}\right)\right] d \tau \\
& +\frac{\beta_{2} T_{4} I_{4}}{\eta_{1}} \int_{0}^{\infty} f_{1}(\tau) e^{-\alpha_{1} \tau}\left[2-\frac{T_{4}}{T}-\frac{T_{\tau} I_{\tau}}{T_{4} I}+\ln \left(\frac{T_{\tau} I_{\tau}}{T I}\right)\right] d \tau \\
& -\frac{\beta_{1} T_{4} V_{4}}{\eta_{2}} \int_{0}^{\infty} f_{2}(\tau) e^{-\alpha_{2} \tau}\left[\frac{V_{4} I_{\tau}}{V I_{4}}-\ln \left(\frac{I_{\tau}}{I}\right)\right] d \tau
\end{aligned}
$$


Hence,

$$
\begin{aligned}
\left.\frac{d L_{4}}{d t}\right|_{(3)}= & -\frac{d}{T}\left(T-T_{4}\right)^{2}-\frac{\beta_{1} T_{4} V_{4}}{\eta_{1}} \int_{0}^{\infty} f_{1}(\tau) e^{-\alpha_{1} \tau}\left[\Phi\left(\frac{T_{4}}{T}\right)+\Phi\left(\frac{T_{\tau} V_{\tau} I_{4}}{T_{4} V_{4} I}\right)\right] d \tau \\
& -\frac{\beta_{2} T_{4} I_{4}}{\eta_{1}} \int_{0}^{\infty} f_{1}(\tau) e^{-\alpha_{1} \tau}\left[\Phi\left(\frac{T_{4}}{T}\right)+\Phi\left(\frac{T_{\tau} I_{\tau}}{T_{4} I}\right)\right] d \tau \\
& -\frac{\beta_{1} T_{4} V_{4}}{\eta_{2}} \int_{0}^{\infty} f_{2}(\tau) e^{-\alpha_{2} \tau} \Phi\left(\frac{V_{4} I_{\tau}}{V I_{4}}\right) d \tau .
\end{aligned}
$$

Thus, $\left.\frac{d L_{4}}{d t}\right|_{(3)} \leq 0$ with equality holds if and only if $T=T_{4}, I=I_{4}$, and $V=V_{4}$. Let

$$
\Gamma=\left\{(T, I, V, W, Z) \mid \frac{d L_{4}}{d t}=0\right\} .
$$

From the second and third equations of the model given by Equation (3), we have

$$
\begin{aligned}
\dot{I} & =\eta_{1}\left(\beta_{1} T_{4} V_{4}+\beta_{2} T_{4} I_{4}\right)-a I_{4}-p I_{4} Z=0, \\
\dot{V} & =k \eta_{2} I_{4}-\mu V_{4}-q V_{4} W=0
\end{aligned}
$$

which implies that $Z=Z_{4}$ and $W=W_{4}$. Then the largest compact invariant set in $\Gamma$ is the singleton $\left\{E_{4}\right\}$. Therefore, $E_{4}$ is globally asymptotically stable.

The conditions of the global stability of $E_{2}$ and those of $E_{3}$ given in (ii) and (iii) of Theorem 4 do not hold simultaneously. In fact, supposing the contrary, then $R_{1}^{W}>1 \geq R_{2}^{Z}$ and $R_{1}^{Z}>1 \geq R_{3}^{W}$. Because $R_{3}^{W} \leq 1$ and $R_{2}^{Z}>\frac{1}{R_{3}^{W}}$, we have $R_{2}^{Z}>1$. This is a contradiction with $R_{2}^{Z} \leq 1$.

According to Equation (12) and Theorem 4, we have the following important result.

Remark 2. Assume $R_{0}>1$.

1. If $\max \left(R_{1}^{W}, R_{1}^{Z}\right) \leq 1$, then Equation (3) converges to $E_{1}$ without immunity.

2. If $\max \left(R_{1}^{W}, R_{1}^{Z}\right)>1$, two cases arise:

(i) When $\max \left(R_{1}^{W}, R_{1}^{Z}\right)=R_{1}^{W}$, the humoral immunity is dominant, and Equation (3) converges to $E_{2}$ if $R_{2}^{Z} \leq 1$ or to $E_{4}$ if $R_{2}^{Z}>1$.

(ii) When $\max \left(R_{1}^{W}, R_{1}^{Z}\right)=R_{1}^{Z}$, the cellular immunity is dominant, and Equation (3) converges to $E_{3}$ without humoral immunity.

From this important remark, we can define the over-domination of humoral immunity when $R_{2}^{Z}>1$ and $R_{3}^{W}>1$ and the over-domination of cellular immunity when $R_{2}^{Z}>1$ and $R_{3}^{W}<1$.

\section{Numerical Simulations}

In this section, we present some numerical simulations in order to validate our theoretical results. For simplicity, we chose $f_{1}(\tau)=\delta\left(\tau-\tau_{1}\right)$ and $f_{2}(\tau)=\delta\left(\tau-\tau_{2}\right)$ with $\tau_{1}$ and $\tau_{2}$ to be the delays in cell infection and virus production, respectively, and $\delta($.$) to be the Dirac function; then our model becomes$

$$
\left\{\begin{aligned}
\dot{T}(t)= & =\lambda-d T(t)-\beta_{1} T(t) V(t)-\beta_{2} T(t) I(t), \\
\dot{I}(t)= & {\left[\beta_{1} T\left(t-\tau_{1}\right) V\left(t-\tau_{1}\right)+\beta_{2} T\left(t-\tau_{1}\right) I\left(t-\tau_{1}\right)\right] e^{-\alpha_{1} \tau_{1}} } \\
& -a I(t)-p I(t) Z(t), \\
\dot{V}(t)= & k I\left(t-\tau_{2}\right) e^{-\alpha_{2} \tau_{2}}-\mu V(t)-q V(t) W(t), \\
\dot{W}(t)= & g V(t) W(t)-h W(t), \\
\dot{Z}(t)= & c I(t) Z(t)-b Z(t) .
\end{aligned}\right.
$$


This system improves the model presented in 2017 by Lin et al. [7], which considered only the humoral immunity and discrete delay in cell infection and ignored the time delay in virus production; that is, $\tau_{2}=0$. In addition, Equation (13) includes many special cases existing in the literature. For example, when $\beta_{2}=0$ and $\tau_{1}=\tau_{2}=0$, we obtain the model presented by Wodarz in [15] and analyzed by Hattaf et al. in [16]. When $\beta_{2}=0$ and $\tau_{2}=0$, we obtain the model of Yan and Wang [17].

The algorithm for the numerical treatment of the delay differential system given by Equation (13) can be derived for the numerical method presented in $[18,19]$. Recently, this numerical method has been used for delayed partial differential equations [20]; it is called the "mixed" Euler method, as it is a mixture of both forward and backward Euler methods. In addition, it is shown that this mixed Euler method preserves the qualitative properties of the corresponding continuous system, such as positivity, boundedness, and global behaviors of solutions. Hence, we discretize the continuous system given by Equation (13) by this numerical method. Thus, we let $\Delta t$ be a time step size and assume that there exist two integers $\left(m_{1}, m_{2}\right) \in \mathrm{N}^{2}$ with $\tau_{1}=m_{1} \Delta t$ and $\tau_{2}=m_{2} \Delta t$. The grids points are $t_{n}=n \Delta t$ for $n \in \mathbf{N}$. By applying the mixed Euler method and using the approximations $T\left(t_{n}\right) \approx T_{n}, I\left(t_{n}\right) \approx I_{n}$, $V\left(t_{n}\right) \approx V_{n}, W\left(t_{n}\right) \approx W_{n}$, and $Z\left(t_{n}\right) \approx Z_{n}$, we obtain the following discrete model:

$$
\left\{\begin{aligned}
T_{n+1}= & T_{n}+\left(\lambda-d T_{n+1}-\beta_{1} T_{n+1} V_{n}-\beta_{2} T_{n+1} I_{n}\right) \Delta t \\
I_{n+1}= & I_{n}+\left(\left[\beta_{1} T_{n-m_{1}+1} V_{n-m_{1}}+\beta_{2} T_{n-m_{1}+1} I_{n-m_{1}}\right] e^{-\alpha_{1} \tau_{1}}\right. \\
& \left.-a I_{n+1}-p I_{n+1} Z_{n}\right) \Delta t \\
V_{n+1}= & V_{n}+\left(k e^{-\alpha_{2} \tau_{2}} I_{n-m_{2}+1}-\mu V_{n+1}-q V_{n+1} W_{n}\right) \Delta t \\
W_{n+1}= & W_{n}+\left(g V_{n+1} W_{n+1}-h W_{n+1}\right) \Delta t \\
Z_{n+1}= & Z_{n}+\left(c I_{n+1} Z_{n+1}-b Z_{n+1}\right) \Delta t
\end{aligned}\right.
$$

where the discrete initial conditions are

$$
\begin{aligned}
& T_{s}=\phi_{1}\left(t_{s}\right), \quad I_{s}=\phi_{2}\left(t_{s}\right), \quad V_{s}=\phi_{3}\left(t_{s}\right), \quad W_{s}=\phi_{4}\left(t_{s}\right), \quad Z_{s}=\phi_{5}\left(t_{s}\right), \\
& \text { for } s \in\{-m,-m+1, \cdots, 0\} \text { and } m=\max \left(m_{1}, m_{2}\right) .
\end{aligned}
$$

The five threshold parameters $R_{0}, R_{1}^{W}, R_{1}^{Z}, R_{2}^{Z}$, and $R_{3}^{W}$ for Equation (13) are given by Equations (7)-(11) with $\eta_{1}=e^{-\alpha_{1} \tau_{1}}$ and $\eta_{2}=e^{-\alpha_{2} \tau_{2}}$. In order to study the impact of cell-to-cell transmission and both arms of adaptive immunity on the HIV dynamics, we chose $\beta_{2}, g$, and $c$ as free parameters. The units of state variables $T, I$, and $Z$ were given by cells $\mu \mathrm{L}^{-1}$. Further, the units of $V$ and $W$ were given by virions $\mu \mathrm{L}^{-1}$ and molecules $\mu \mathrm{L}^{-1}$, respectively. The other parameter values for the simulation are listed in Table 1. 
Table 1. List of parameters.

\begin{tabular}{|c|c|c|c|c|}
\hline Parameter & Unit & Value & Range & Source \\
\hline$\lambda$ & cells $\mu \mathrm{L}^{-1}$ day $^{-1}$ & 10 & $5.9770-24.1860$ & [21] \\
\hline$d$ & day $^{-1}$ & 0.0139 & - & [22] \\
\hline$\beta_{1}$ & $\mu \mathrm{L}$ virion ${ }^{-1}$ day $^{-1}$ & $2.4 \times 10^{-5}$ & $2.4 \times 10^{-5}-4.8 \times 10^{-3}$ & {$[3,23]$} \\
\hline$a$ & day $^{-1}$ & 0.29 & $0.2666-0.7073$ & {$[3,24,25]$} \\
\hline$\alpha_{1}$ & day $^{-1}$ & 0.01 & - & [26] \\
\hline$\mu$ & day $^{-1}$ & 3 & $2.06-3.81$ & [3] \\
\hline$k$ & virion cell $^{-1}$ day $^{-1}$ & 50 & $27-7073$ & [21] \\
\hline$\alpha_{2}$ & day $^{-1}$ & 0.01 & - & [26] \\
\hline$p$ & cell $^{-1} \mu \mathrm{L}$ day $^{-1}$ & 0.001 & $0.001-1$ & [27-30] \\
\hline$q$ & molecule $^{-1} \mu \mathrm{L}_{\text {day }}{ }^{-1}$ & 0.5 & - & Assumed \\
\hline$h$ & day $^{-1}$ & 0.5 & - & Assumed \\
\hline$b$ & day $^{-1}$ & 0.1 & $0.05-0.15$ & {$[28,29]$} \\
\hline$\tau_{1}$ & days & 1.5 & $0-2$ & [31] \\
\hline$\tau_{2}$ & days & 0.5 & - & [32] \\
\hline
\end{tabular}

For the case in which $\beta_{2}=10^{-6}, g=10^{-5}$, and $c=0.002$, we obtained $R_{0}=0.9751$. It follows from Theorem 2 that Equation (13) has one infection-free equilibrium $E_{0}(719.4245,0,0,0,0)$. From Figure 1, we see that the concentration of uninfected $\mathrm{CD}^{+}{ }^{+} \mathrm{T}$ cells increased and tended to the value $T_{0}=719.4245$, while the concentrations of infected cells, free HIV particles, antibodies, and CTL cells decreased and tended to zero. This means that $E_{0}$ is globally asymptotically stable and that the virus will be cleared. This confirms the result in Theorem 3.

For the case in which $\beta_{2}=1.5 \times 10^{-4}, g=10^{-5}$, and $c=0.002$, we obtained $R_{0}=1.3392$, $R_{1}^{W}=0.0143$, and $R_{1}^{Z}=0.1721$. It follows that case $(i)$ of Theorem 4 occurs, and the infection equilibrium without immunity $E_{1}(537.9692,8.5643,142.0261,0,0)$ is globally asymptotically stable (see Figure 2).

For the case in which $\beta_{2}=3.4 \times 10^{-4}, g=10^{-3}$, and $c=0.002$, we obtained $R_{0}=1.8036$, $R_{1}^{W}=2.5099$, and $R_{2}^{Z}=0.2000$. From (ii) of Theorem 4 , the infection equilibrium with only humoral immunity $E_{2}(507.5951,10.0014,99.9785,3.9525,0)$ is globally asymptotically stable (see Figure 3).

For the case in which $\beta_{2}=5.4 \times 10^{-4}, g=10^{-3}$, and $c=0.02$, we obtained $R_{0}=2.2923$, $R_{1}^{Z}=3.8301$, and $R_{3}^{W}=0.8292$. By ( $\left.i i i\right)$ of Theorem 4 , the infection equilibrium with only cellular immunity $E_{3}(537.3806,5.0015,83.1539,0,211.6047)$ is globally asymptotically stable (see Figure 4).

For the case in which $\beta_{2}=5.4 \times 10^{-4}, g=10^{-2}$, and $c=0.02$, we obtained $R_{0}=2.2923$, $R_{2}^{Z}=1.8783$, and $R_{3}^{W}=8.2918$. Using (iv) of Theorem 4 , we deduce that the infection equilibrium with both arms of immunity $E_{4}(594.0271,4.9769,10.0246,43.3843,53.3821)$ is globally asymptotically stable (see Figure 5). 

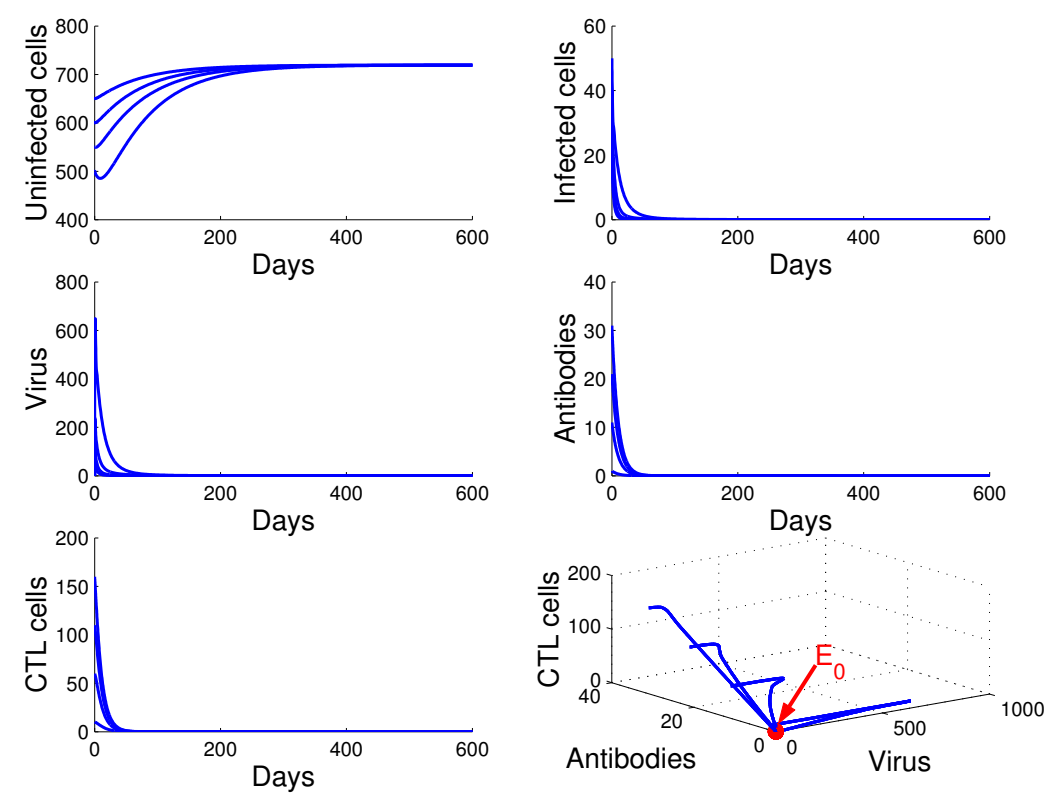

Figure 1. Demonstration of the global stability of the infection-free equilibrium $E_{0}$ for $R_{0}=0.9751 \leq 1$.
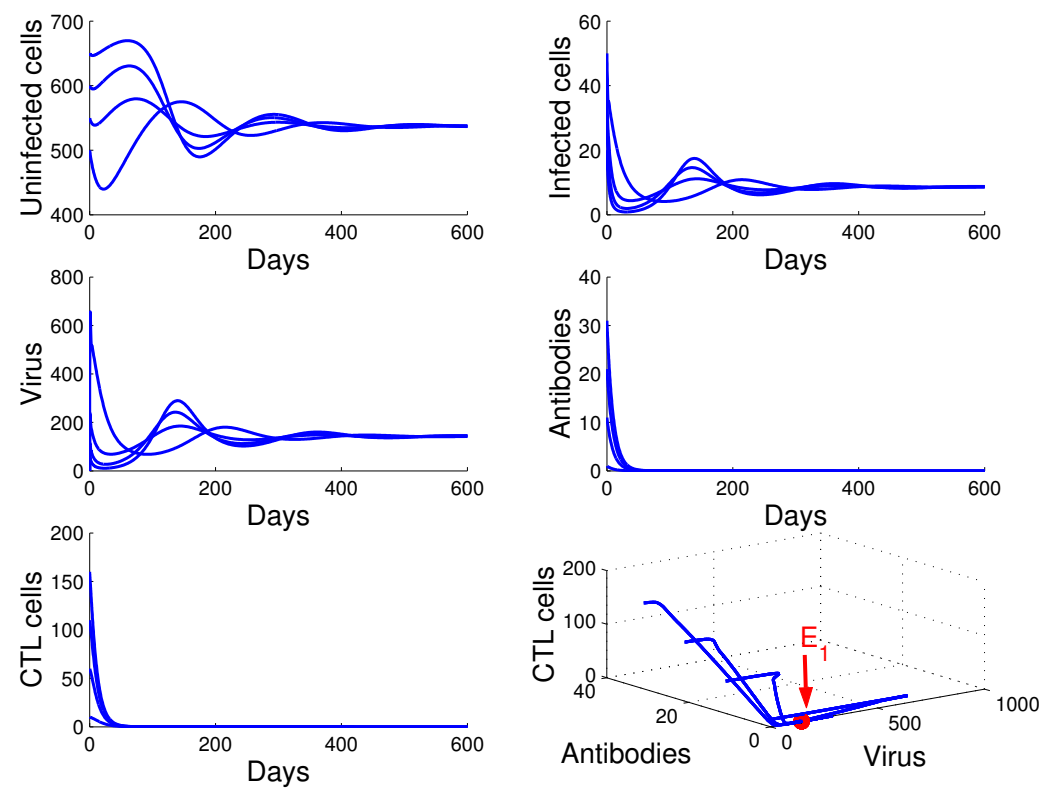

Figure 2. Demonstration of the global stability of the infection equilibrium without immunity $E_{1}$ for $R_{0}=1.3392>1, R_{1}^{W}=0.0143 \leq 1$, and $R_{1}^{Z}=0.1721 \leq 1$. 

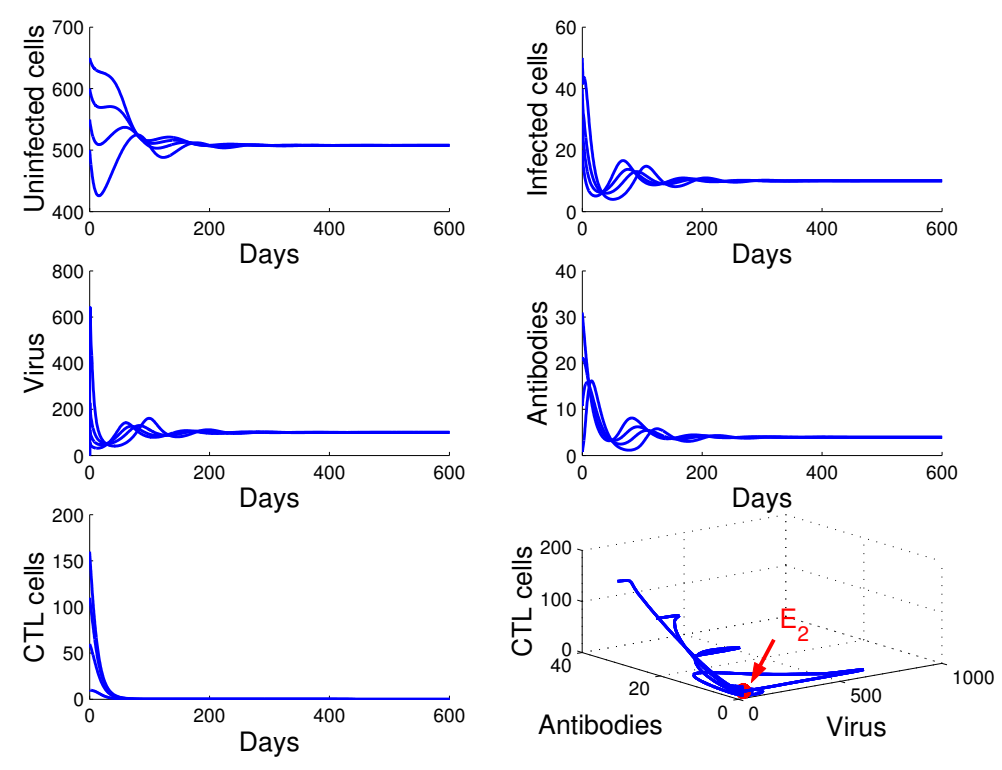

Figure 3. Demonstration of the global stability of the infection equilibrium with only humoral immunity $E_{2}$ for $R_{0}=1.8036>1, R_{1}^{W}=2.5099>1$, and $R_{2}^{Z}=0.2000 \leq 1$.
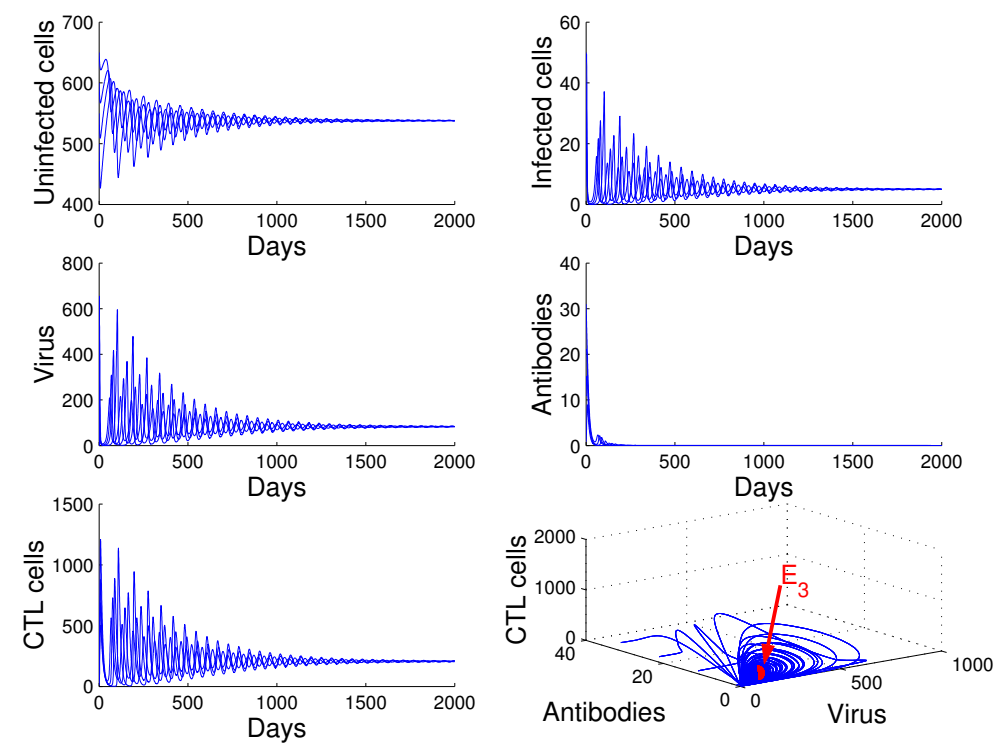

Figure 4. Demonstration of the global stability of the infection equilibrium with only cellular immunity $E_{3}$ for $R_{0}=2.2923>1, R_{1}^{Z}=3.8301>1$, and $R_{3}^{W}=0.8292 \leq 1$. 

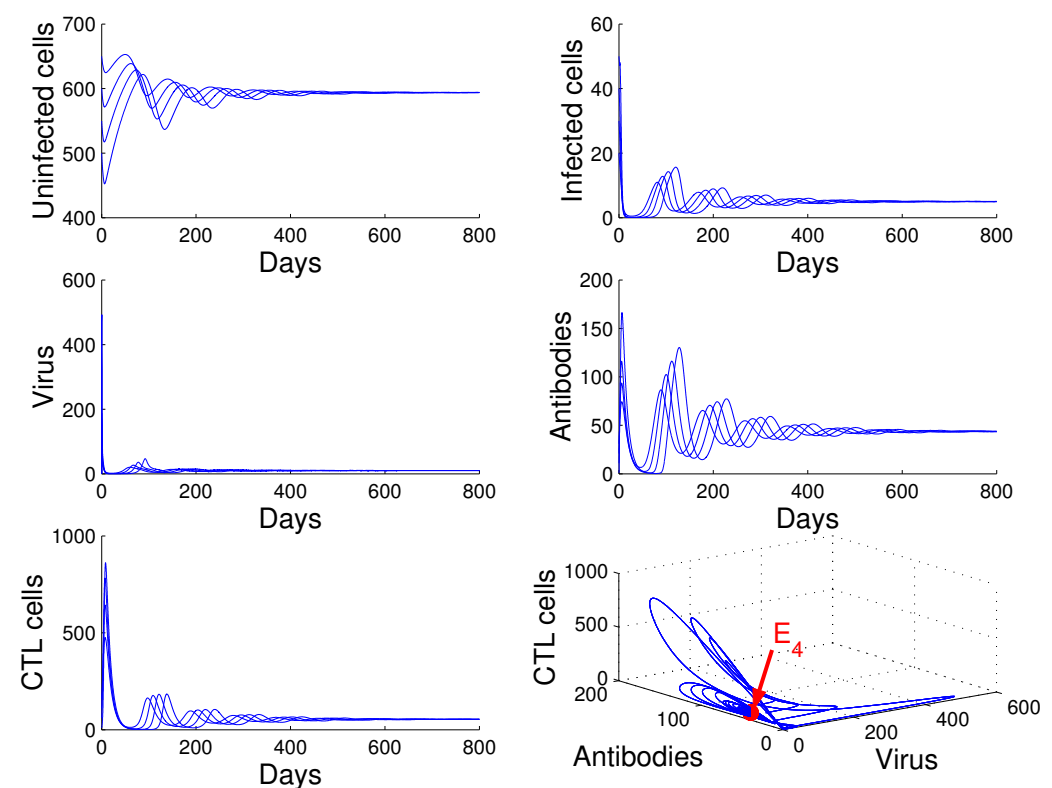

Figure 5. Demonstration of the global stability of the infection equilibrium with both arms of immunity $E_{4}$ for $R_{0}=2.2923>1, R_{2}^{Z}=1.8783>1$, and $R_{3}^{W}=8.2918>1$.

\section{Conclusions}

In this paper, we have modeled the role of the adaptive immunity in HIV infection by proposing a new mathematical model that takes into account the classical virus-to-cell infection, the direct cell-to-cell transmission, and the two kinds of delays during infection processes and virus production. By a rigorous mathematical analysis, we have proved that the global dynamics of the proposed model is fully determined by five threshold parameters, which are the basic reproduction number $R_{0}$, and the reproduction numbers for humoral immunity $R_{1}^{W}$, for cellular immunity $R_{1}^{Z}$, for cellular immunity in competition $R_{2}^{Z}$, and for humoral immunity in competition $R_{3}^{W}$. More precisely, the infection-free equilibrium is globally asymptotically stable if $R_{0} \leq 1$, which biologically means that the HIV is cleared and the infection dies out. When $R_{0}>1$, our model has four infection equilibria, which are the following: (i) the infection equilibrium without immunity is globally asymptotically stable if $R_{1}^{W} \leq 1$ and $R_{1}^{Z} \leq 1$; (ii) the infection equilibrium with only humoral immunity is globally asymptotically stable if $R_{1}^{W}>1$ and $R_{2}^{Z} \leq 1$; (iii) the infection equilibrium with only cellular immunity is globally asymptotically stable if $R_{1}^{Z}>1$ and $R_{3}^{W} \leq 1$; (iv) the infection equilibrium with both cellular and humoral immune responses is globally asymptotically stable if $R_{2}^{Z}>1$ and $R_{3}^{W}>1$. Biologically, this implies that the HIV persists and the infection becomes chronic when the basic reproduction number is greater than 1. Additionally, the activation of one or both arms of immunity is unable to eliminate the virus in the human body.

From Remark 2, we can deduce that the over-domination of cellular immunity leads to the absence of the humoral immunity, and the over-domination of the humoral immunity leads to the persistence of HIV infection with a weak response of both arms of immunity. This biological result may be an explanation for the dysfunction of the adaptive immunity in HIV-infected patients. Our model can be adapted for HBV infection, and the above result can also explain the dysfunction of the adaptive immune response in patients infected with HBV, which is still largely incomplete [33].

Furthermore, the model and the results presented in this study improve and generalize the models and the corresponding results in more recent papers with only cellular immunity [5], with only humoral immunity [6-9], and with both arms of immunity [15,17]. 
Author Contributions: Both authors contributed equally to the writing of this paper. They both read and approved the final version of the manuscript.

Acknowledgments: We would like to thank the editor and anonymous referees for their very helpful comments and suggestions that greatly improved the quality of this study.

Conflicts of Interest: The authors declare no conflict of interest.

\section{References}

1. WHO. HIV/AIDS, Fact Sheet, July 2017. Available online: http://www.who.int/mediacentre/factsheets / fs360/en/ (accessed on 8 May 2018).

2. Royaume du Maroc, Mise en oeuvre de la déclaration politique sur le VIH/sida, Rapport National 2015. Available online: http:/ / www.unaids.org/sites/default/files/country/documents/MAR_narrative_report_ 2015.pdf (accessed on 8 May 2018).

3. Perelson, A.S.; Neumann, A.U.; Markowitz, M.; Leonard, J.M.; Ho, D.D. HIV-1 dynamics in vivo: Virion clearance rate, infected cell life-span, and viral generation time. Science 1996, 271, 1582-1586. [CrossRef] [PubMed]

4. Lai, X.; Zou, X. Modeling HIV-1 virus dynamics with both virus-to-cell infection and cell-to-cell transmission. SIAM J. Appl. Math. 2014, 74, 898-917. [CrossRef]

5. Wang, J.; Guo, M.; Liu, X.; Zhao, Z. Threshold dynamics of HIV-1 virus model with cell-to-cell transmission, cell-mediated immune responses and distributed delay. Appl. Math. Comput. 2016, 291, 149-161. [CrossRef]

6. Elaiw, A.M.; Raezah, A.A.; Alofi, A.S. Effect of humoral immunity on HIV-1 dynamics with virus-to-target and infected-to-target infections. AIP Adv. 2016, 6, 085204. [CrossRef]

7. Lin, J.; Xu, R.; Tian, X. Threshold dynamics of an HIV-1 virus model with both virus-to-cell and cell-to-cell transmissions, intracellular delay, and humoral immunity. Appl. Math. Comput. 2017, 315, 516-530. [CrossRef]

8. Wang, S.F.; Zou, D.Y. Global stability of in-host viral models with humoral immunity and intracellular delays. Appl. Math. Model. 2012, 36, 1313-1322. [CrossRef]

9. Murase, A.; Sasaki, T.; Kajiwara, T. Stability analysis of pathogen-immune interaction dynamics. J. Math. Biol. 2005, 51, 247-267. [CrossRef] [PubMed]

10. Hale, J.K.; Kato, J. Phase space for retarded equations with infinite delay. Funkcialaj Ekvacioj 1978, $21,11-41$.

11. Hino, Y.; Murakami, S.; Naito, T. Functional Differential Equations with Infinite Delay; Lecture Notes in Mathematics; Springer: Berlin, Germany, 1991; Volume 1473.

12. Kuang, Y. Delay Differential Equations with Applications in Population Dynamics; Academic Press: San Diego, CA, USA, 1993.

13. Hattaf, K.; Yousfi, N. A generalized virus dynamics model with cell-to-cell transmission and cure rate. Adv. Differ. Equ. 2016, 2016, 174. [CrossRef]

14. Hale, J.K.; Lunel, S.M.V. Introduction to Functional Differential Equations; Springer: New York, NY, USA, 1993.

15. Wodarz, D. Hepatitis C virus dynamics and pathology: The role of CTL and antibody responses. J. Gen. Virol. 2003, 84, 1743-1750. [CrossRef] [PubMed]

16. Yousfi, N.; Hattaf, K.; Rachik, M. Analysis of a HCV model with CTL and antibody responses. Appl. Math. Sci. 2009, 3, 2835-2845.

17. Yan, Y.; Wang, W. Global stability of a five-dimensionalmodel with immune responses and delay. Discret. Contin. Dyn. Syst. Ser. B 2012, 1, 401-416.

18. Hattaf, K.; Yousfi, N. Global properties of a discrete viral infection model with general incidence rate. Math. Methods Appl. Sci. 2016, 39, 998-1004. [CrossRef]

19. Hattaf, K.; Yousfi, N. A numerical method for a delayed viral infection model with general incidence rate. J. King Saud Univ. Sci. 2016, 28, 368-374. [CrossRef]

20. Hattaf, K.; Yousfi, N. A numerical method for delayed partial differential equations describing infectious diseases. Comput. Math. Appl. 2016, 72, 2741-2750. [CrossRef]

21. Maziane, M.; Lotfi, E.M.; Hattaf, K.; Yousfi, N. Dynamics of a class of HIV infection models with cure of infected cells in eclipse stage. Acta Biotheor. 2015, 63, 363-380. [CrossRef] [PubMed] 
22. Bourgeois, C.; Hao, Z.; Rajewsky, K.; Potocnik, A.; Stockinger, B. Ablation of thymic export causes accelerated decay of naive CD4 T cells in the periphery because of activation by environmental antigen. Proc. Natl. Acad. Sci. USA 2008, 105, 8691-8696. [CrossRef] [PubMed]

23. Stafford, M.; Corey, L.; Cao, Y.; Daar, E.; Ho, D.; Perelson, A. Modeling plasma virus concentration during primary HIV infection. J. Theor. Biol. 2000, 203, 285-301. [CrossRef] [PubMed]

24. Wei, X.; Ghosh, S.K.; Taylor, M.E.; Johnson, V.A.; Emini, E.A.; Deutsch, P.; Lifson, J.D.; Bonhoeffer, S.; Nowak, M.A.; Hahn, B.H.; et al. Viral dynamics in human immunodeficiency virus type 1 infection. Nature 1995, 373, 117-122. [CrossRef] [PubMed]

25. Ho, D.D.; Neumann, A.U.; Perelson, A.S.; Chen, W.; Leonard, J.M.; Markowitz, M. Rapid turnover of plasma virions and CD4 lymphocytes in HIV-1 infection. Nature 1995, 373, 123-126. [CrossRef] [PubMed]

26. Miao, H.; Teng, Z.; Li, Z. Global Stability of Delayed Viral Infection Models with Nonlinear Antibody and CTL Immune Responses and General Incidence Rate. Comput. Math. Methods Med. 2016, 2016, 3903726. [CrossRef] [PubMed]

27. Arnaout, R.; Ramy, A.; Wodarz, D. HIV-1 dynamics revisited: biphasic decay by cytotoxic T lymphocyte killing? Proc. R. Soc. Lond. B Biol. Sci. 2000, 267, 1347-1354. [CrossRef] [PubMed]

28. Culshaw, R.V.; Ruan, S.; Spiteri, R.J. Optimal HIV treatment by maximising immune response. J. Math. Biol. 2004, 48, 545-562. [CrossRef] [PubMed]

29. Nowak, M.A.; Bangham, C. Population dynamics of immune response to persistent viruses. Science 1996, 272, 74-79. [CrossRef] [PubMed]

30. Wang, Y.; Zhou, Y.; Brauer, F.; Heffernan, J. Viral dynamics model with CTL immune response incorporating antiretroviral therapy. J. Math. Biol. 2013, 67, 901-934. [CrossRef] [PubMed]

31. Wang, Y.; Zhou, Y.; Wu, J.; Heffernan, J. Oscillatory viral dynamics in a delayed HIV pathogenesis model. Math. Biosci. 2009, 219, 104-112. [CrossRef] [PubMed]

32. Zhu, H.; Zou, X. Impact of delays in cell infection and virus production on HIV-1 dynamics. Math. Med. Biol. 2008, 25, 99-112. [CrossRef] [PubMed]

33. Boni, C.; Fisicaro, P.; Valdatta, C.; Amadei, B.; di Vincenzo, P.; Giuberti, T.; Laccabue, D.; Zerbini, A.; Cavalli, A.; Missale, G.; et al. Characterization of HBV-specific T cell dysfunction in chronic HBV infection. J. Virol. 2007, 8, 4215-4225. [CrossRef] [PubMed]

(C) 2018 by the authors. Licensee MDPI, Basel, Switzerland. This article is an open access article distributed under the terms and conditions of the Creative Commons Attribution (CC BY) license (http:/ / creativecommons.org/licenses/by/4.0/). 